\title{
Facile and Controllable Synthesis N-doping Porous Graphene for High-Performance Supercapacitor
}

Xiuxian He ${ }^{a \dagger}$, Zheng Tang ${ }^{a \dagger}$, LianLian Gao $^{a}$, Fangyuan Wang $^{a}$, Jinping Zhao $^{a}$ *, Zhichao Miao ${ }^{a}$, Xiaozhong $W^{a}$, Jin Zhou ${ }^{a}$, Yang Su ${ }^{b}$, Shuping Zhuo *

${ }^{\text {a }}$ School of Chemistry and Chemical Engineering, Shandong University of Technology, Zibo 255049, P. R. China

${ }^{\mathrm{b}}$ Department of Materials, Loughborough University, Loughborough LE11 3TU, UK

$\dagger$ These authors contribute equally to this paper

*Corresponding author

E-mail: jpzhao@sdut.edu.cn; zhuosp_academic@yahoo.com 



\section{Abstract}

Engineering graphene with efficient ion and electron transport properties is vital for development of high performance, next generation energy storage devices. The protocols generally involve pore generation and doping of graphene with the electrondonating/accepting atoms. However, the reported protocols are proven to be either complicated, or lack of precise process control. Herein we report the realization of pore generation ,control, and doping ( $\mathrm{N}$-doping) of graphene in one single process using environment friendly $\mathrm{N}_{2}$ gas as doping agent by a thermal treatment of metal salt infiltrated graphene nanosheets, the metal ions are reduced during the thermal process and act as an etchant to generate pores and promotes atomization of $\mathrm{N}_{2}$ for efficient $\mathrm{N}$ doping. Furthermore, we found that the pore size and density could be controlled by choosing different metal salts, which allows us to investigate the influence of pore structure of graphene nanosheets in their supercapacitor performance. Specifically,the $\mathrm{N}$-doped, porous graphene $(\mathrm{PG})$ shows an average pore size of $\sim \mathrm{xx} \mathrm{nm}$, and a pore density $\mathrm{xx}$, the The as-prepared PG-Ni exhibits high specific capacitance of $575 \mathrm{~F} / \mathrm{g}$ along with the energy storage of $51.2 \mathrm{Wh} / \mathrm{kg}$ at $0.5 \mathrm{~A} / \mathrm{g}$ current density. The PG also shows good rate, cycling performances and could retain $90.3 \%$ of its initial capacitance at a current density of $2 \mathrm{~A} / \mathrm{g}$ even after 1000 cycles. Considering the excellent performance, ease of the process, and pore structure controllability, we believe the method provides a new route for developing graphene based high performance supercapacitors. 


\section{Introduction}

Supercapacitors (SCs) are promising and efficient energy storage devices that continue to attract increasing attentions owning to their high power density, exceptional cycle life, and low maintenance cost[1-3]. However, the energy storage density in supercapacitors is currently an order of magnitude lower than that of batteries, for example, the energy density of existing supercapacitor devices, which are mainly based on porous activated carbon (AC), is about 4 to $5 \mathrm{Wh} \mathrm{kg}^{-1}$, whereas that of lead acid batteries is in the range of 26 to $34 \mathrm{Wh} \mathrm{kg}^{-1}[4]$. Therefore, one of the great challenges for the next generation supercapacitoror is to obtain the high energy density while maintaining its superior cycling performance. Graphene-based materials which have large surface area and extraordinary electrical conductivity offer a high theoretical capacitance of $550 \mathrm{~F} / \mathrm{g}[5-8]$. However, the experimentally achieved capacitance of graphene-based SCs is much lower than the theoretical capacitance $[9,10]$. This is due to the strong $\pi-\pi$ interactions and Van der Waals interactions among the graphene single layers, the graphene sheets tend to restack[11, 12]. It leads to a significant decrease in surface area, therefore, itcould not provide adequate electrolyte ionic conduction during energy storage process(或者说 charge-discharge cycle/process?). To increase the surface area and generate channels for ion transfer are important strategies to improve the performance of graphene-based supercapacitor. Porous graphene (PG), which is a derivative of graphene with nanoscale pores on the sheets, is reported to have significant higher capacitance comparing to pristine graphene because their higher 
surface area and faster electron and ion transport[13-17]. The main methods currently utilized to prepare PG are, plasma etching[18], chemical vapor deposition[19], ion irradiation[20], steam-etching[21], lithography[22] and ultrasonication of graphene oxide (GO) under harsh acidic or alkaline condition[23, 24]. However, the most preparation methods are either inefficient or lack of controlling of the pore structure which is very important for the supercapacitor applications.

The other alternative strategy to boost the capacitance of graphene-based supercapacitor is doping with heteroatoms. For example, N-doping of graphene, with nitrogen atoms replacing carbon atoms, is reported to increase the electronic conductivity and wettability of graphene nanosheets, which are beneficial for the electron and electrolyte ion transport during the energy storage process[25]. Further, Ndoping lso introduces pseudo-capacitance in carbons materials to further improve the capacitance of the graphene electrode[26, 27]. During the nitrogen configurations (pyridinic, pyrrolic and pyridone groups), the basal-plane pyridinic $\mathrm{N}$ was found to have a dominant role in the capacitance enhancement $[9,28]$. Therefore, controlling the appropriate nitrogen configuration can greatly improve the capacitance of N-doping graphene electrodes.

It is reasonable to expect that if the graphene nanosheets could be engineered to be porous, in the same time, $\mathrm{N}$-doping, a synergetic effects should arise, and their capacitance in supercapacitor would be much improved. However, such studies generally need a two-step synthesis to accomplish the pore generation and N-doping, further, the doping agent normally involves toxic $\mathrm{NH}_{3}$, which could potentially raise 
the environmental concerns(师姐, 有文献么? ). Here, we report the one-step synthesis of porous, $\mathrm{N}$-doping graphene nanosheets by $\mathrm{N}_{2}$ gas, the idea is inspired by the phenomenon that metal nanoparticles could etch the graphene into the porous structure with nanoscale precision via catalytic hydrogenation process[29-33]. During the process, defective sites generated on the pore edges are employedfor N-doping simultaneously. In detail, the metal salt absorbed on graphene oxide (GO) nanosheets was thermally annealed in the inert $\mathrm{N}_{2}$ gas at a temperature of $800{ }^{\circ} \mathrm{C}$, we found that the thermal treatment converts the metal salt to metal nanoparticles, which etch the graphene sheets to form porous graphene nanosheet and in the same time, surprisingly, we found the metal nanoparticles catalytically decompose the $\mathrm{N}_{2}$ into the active $\mathrm{N}$ atoms, which then bind to the defective sites generated during etching process, we therefore, achieve the pore generation and $\mathrm{N}$-doping in one step using environment friendly $\mathrm{N}_{2}$ gas source. In addition, we found the porous structure (pore size and density), the dopant bonding configuration and doping amount can be controlled by change the metal salt and nitrogen source. Finally, by applying the N-doping PG as the supercapcitor electrodes, the supercapacitor shows a reversible specific capacity as high as 575 F/g, excellent cyclability and rate capability, suggesting PG nanosheets arepromising material for high-performance supercapacitor applications. 


\section{Experimental}

\subsection{Materials}

Flake graphite (99\%, 32 mesh), which was purchased from Qingdao Tianheda Graphite Ltd. Co (Qingdao, China). $\mathrm{H}_{2} \mathrm{SO}_{4}$ (purity $\geq 98.0 \%$ ), $\mathrm{KMnO}_{4}$ (purity $\geq 99.5 \%$ ), Co $\left(\mathrm{NO}_{3}\right)_{2} \cdot 6 \mathrm{H}_{2} \mathrm{O}$ (Aladdin Co., Ltd, $\left.\mathrm{H} 1507036\right)$ and $\mathrm{Ni}\left(\mathrm{NO}_{3}\right)_{2} \cdot 6 \mathrm{H}_{2} \mathrm{O}$ (Aladdin Co., Ltd, N140967) were obtained from Aladdin Chemicals Co., Ltd. (Shanghai, China). All reagents were used as received without further purification.

\subsection{Preparation of GO}

GO was prepared from flake graphite by the modified Hummers method shown in previous work[34]. The flake graphite $(2 \mathrm{~g})$ was added into concentrated $\mathrm{H}_{2} \mathrm{SO}_{4}$ (96 $\mathrm{mL}$ ) under stirring at $0{ }^{\circ} \mathrm{C}$, followed by the slow addition of $\mathrm{KMnO}_{4}(12 \mathrm{~g})$. The mixture was stirred in sequence at $0,35,75{ }^{\circ} \mathrm{C}$ for 90,150 and $90 \mathrm{~min}$, respectively. Subsequently, deionized water $(80 \mathrm{~mL})$ was added dropwise under vigorous stirring. Afterwards, $200 \mathrm{~mL}$ deionized water and $12 \mathrm{~mL} \mathrm{H}_{2} \mathrm{O}_{2}$ solution (30\%) were added under stirring condition for additional $10 \mathrm{~min}$. The resulting graphite oxide was washed repeatedly by a large amount of deionized water to $\mathrm{pH}$ at 6-7.

To obtain the GO suspension, the above graphite oxide slurry was diluted with water in a volume ratio of 1:10 and mildly sonicated for $30 \mathrm{~min}$. Then, the dispersion was centrifuged to remove the visible particles. Finally,the supernatant is collected anddiluted with deionized water to a concentration of $1 \mathrm{mg} / \mathrm{mL}$. 


\subsection{Preparation of N-doping PG}

PG was synthesized by using different nitrate melt $(\mathrm{m}=\mathrm{Co}, \mathrm{Ni})$. Briefly, $50 \mathrm{mg}$ $\mathrm{Ni}\left(\mathrm{NO}_{3}\right)_{2}$ was added into $100 \mathrm{~mL}$ GO suspension. And the mixture was sonicated for $30 \mathrm{~min}$ and stirred for $12 \mathrm{~h}$. The final mixture was quickly frozen by liquid nitrogen and freeze-dried to obtained the $\mathrm{GO}-\mathrm{Ni}\left(\mathrm{NO}_{3}\right)_{2}$ powder. The powder was heated from room temperature to $800{ }^{\circ} \mathrm{C}$ at a rate of $10{ }^{\circ} \mathrm{C} / \mathrm{min}$ in a tube furnace and held at this temperature for $2 \mathrm{~h}$ under a flowing $\mathrm{N}_{2}$ gas. The obtained PG with $\mathrm{Ni}$ particles (designated as $\mathrm{PG} / \mathrm{Ni})$ was immersed in diluted $\mathrm{HCl}(0.1 \mathrm{~mol} / \mathrm{L})$ and stirred for 2 days to remove the Ni particles. Finally, the resulting sample was collected by filtration, washing with water and ethanol, and freeze-dried for $24 \mathrm{~h}$, that is $\mathrm{N}$-doping PG,designated as PG-Ni (remove Ni particles). As a comparison, in order to investigate the effect of different metals on pore structure and $\mathrm{N}$-doping configuration, $\mathrm{Co}\left(\mathrm{NO}_{3}\right)_{2}$ was used to prepare PG with the same procedure. The finally obtained $\mathrm{N}$-doping PG was designated as PG-Co.

In order to explore the effect of different nitrogen sources on the nitrogen doping content and configuration, the urea was used as the nitrogen source. PG precursors were prepared via the same process as PG-Ni except the heating process. In this heating process, Ar gas was used instead of $\mathrm{N}_{2}$ gas to avoid the $\mathrm{N}$-doping, and the obtained $\mathrm{PG}$ was mixed with urea, and heated to $800{ }^{\circ} \mathrm{C}$ at a rate of $10{ }^{\circ} \mathrm{C} / \mathrm{min}$ in a tube furnace and held at this temperature for $2 \mathrm{~h}$ under Ar gas. 


\subsection{Characterization}

The morphology and structure of samples were characterized by scanning electron microscopy (SEM, Sirion 200FEI). X-ray diffraction (XRD) patterns were collected on a Bruker D8 Advance X-ray diffractometer using a $\mathrm{Cu} K \alpha$ source $(\lambda=0.154056 \mathrm{~nm})$. Transmission electron microscopy (TEM) images were taken on a JEOL-2100 microscope at an acceleration voltage of $200 \mathrm{kV}$. Raman spectroscopy analysis was performed on a Renishaw via Reflex Raman microscope using $532 \mathrm{~nm}$ green laser excitation. The N-doping structure was also investigated by X-ray photoelectron spectroscopy (XPS) Escalab 250). The specific surface area and pore size distribution of the samples were calculated based on the measurement of the Brunauer-EmmettTeller (BET).

\subsection{Electrochemical measurements}

The electrochemical tests were carried out on a CHI660D electrochemical workstation (Chenhua Instruments Co. Ltd, Shanghai) using a traditional three electrodes system in $1.0 \mathrm{~mol} / \mathrm{L} \mathrm{KOH}$ electrolyte at room temperature. Here, a saturated calomel electrode and a platinum foil are used as reference electrode and counter electrode respectively, and a nickel foil loaded with N-doping PG is used as the working electrode.

Briefly, The electrode active material, acetylene black (Super-P) and polytetrafluoroethylene (PTFE) are added dropwise with an appropriate amount of 
absolute ethanol with a mass ratio of 80:10:10, and then thoroughly ground into a uniform paste, then dried in vacuum at $80{ }^{\circ} \mathrm{C}$ for $12 \mathrm{~h}$ to remove the solvent. The obtained mixture was first cut into slices $\left(2 \mathrm{mg}, 1 \times 1 \mathrm{~cm}^{2}\right)$. The working electrodes were prepared by pressing the $1 \mathrm{mg}$ slices onto nickel foam $\left(1 \times 3 \mathrm{~cm}^{2}\right)$ under $8 \mathrm{MPa}$ for 30 s. The electrodes were then soaked in electrolyte for $2 \mathrm{~h}$ before measurements.

The cyclic voltammetry $(\mathrm{CV})$ tests were measured under different scan rates from 5.0 to $100.0 \mathrm{mV} / \mathrm{s}$ in the range of $-0.8 \sim 0.0 \mathrm{~V}$. The galvanostatic charge-discharge (GCD) curves were obtained at different current densities (from 0.5 to $10.0 \mathrm{~A} / \mathrm{g}$ ) at the same potential range as $\mathrm{CV}$ measurements, and the electrochemical impedance spectroscopy (EIS) tests were carried out in the frequency range from $100 \mathrm{kHz}$ to $10 \mathrm{mHz}$ with the potential amplitude of $5 \mathrm{mV}$. The cycling life was measured at $2 \mathrm{~A} / \mathrm{g}$ from $-0.8 \sim 0.0 \mathrm{~V}$ for 1000 cycles.

The specific capacitance (C), power density (P) and energy density (E) were calculated from the equations:

$$
\begin{aligned}
\mathrm{C} & =\frac{I}{(d E / d t) \times m}(F / g) \\
\mathrm{E} & =\frac{1}{8} C V^{2} \\
\mathrm{P} & =\frac{E}{\Delta t}
\end{aligned}
$$

where I is the discharge current (A), $t$ is the discharge time (s), V is the voltage change (V) during the discharge process, and $\mathrm{m}$ is the mass of the corresponding electrode material $(\mathrm{g})$. 


\section{Results and Discussion}

\subsection{Structural characterization}
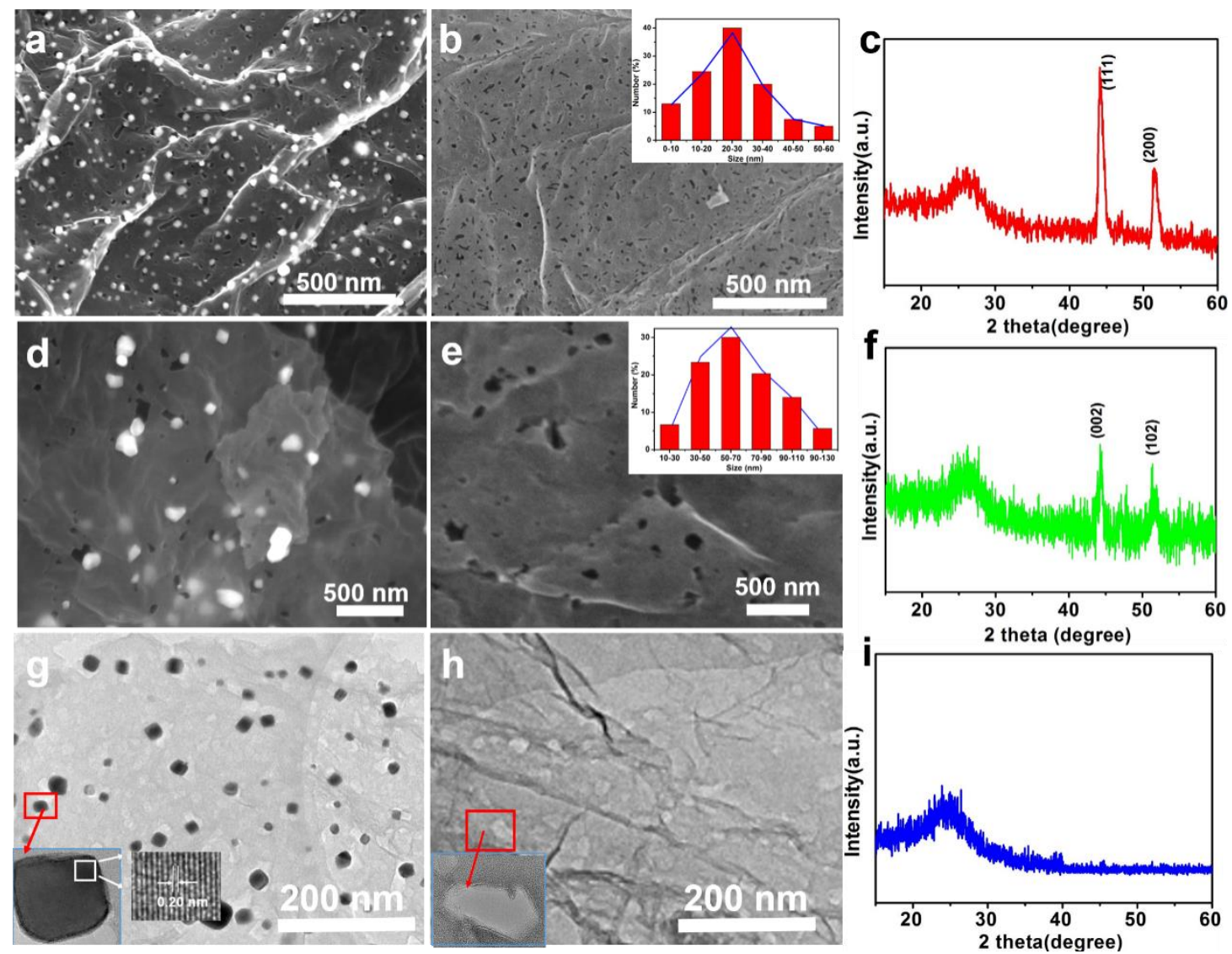

Fig.1. a. SEM image of PG/Ni; b. SEM image of PG-Ni; the inset is size distribution histograms of pores of PG-Ni; c. XRD pattern of PG/Ni; d. SEM image of PG/Co; e. SEM image of PG-Co; the inset is size distribution histograms of pores of PG-Co; f. XRD pattern of PG/Co; g. TEM image of PG/Ni; h.TEM image of PG-Ni; i. XRD pattern of PG-Ni.

To examine the effectiveness of the pore generation on the graphene sheets, different PG materials are examined by the SEM. Fig. S1 displays the typical SEM image of the reduced GO, it shows 2D sheet structure and no pore observed on the graphene sheet. After the thermal treatment with the metal salt under the $\mathrm{N}_{2}$, we observed the formation 
of nanoparticles (Fig.1a and 1d), the XRD analysis shows these nanoparticles are metallic, for example, as shown in Fig. 1c and 1f, when the PG/Ni and PG/Co are used for the synthesis, the metallic Ni and Co nanoparticles are obtained respectively. This is confirmed bythe XRD peaks corresponding to metallic Ni and Co respectively . This results also in accordance with the reference[33]. To examine whether the pore generation is successful, we examine the samples under the SEM, it is found that, at the vicinity of each nanoparticle, the nanopores are formed as expected (Fig. 1a and 1d). To analyze the pore structure, we have leached the nanoparticles by $\mathrm{HCl}$ to obtain the PG. The pore size is measured based on the SEM images. Comparing the pore size generated with $\mathrm{Ni}$ and $\mathrm{Co}$ nanoparticles, we found that $\mathrm{Ni}$ nanoparticles result in an average pore size at $22.4 \mathrm{~nm}$, and a very high pore density, while in the case of Co, the average pore size is $70.6 \mathrm{~nm}$, the pore density is significantly lower than that of $\mathrm{Ni}$. This indicates that different metal particles have great impact on the pore generation process. To better understand the pore generation process, we have observed our samples under the TEM, we found that during the etching process, the amorphous carbon can form around particles (Fig. 1g). When the amorphous carbon reaches to the critical thickness that the particle lose etching activity and stop moving, the pore generation terminates[33]. Ni melt can generate smaller and more particles on graphene sheet than that of Co melt, therefore, enables a better pore generation capacity 

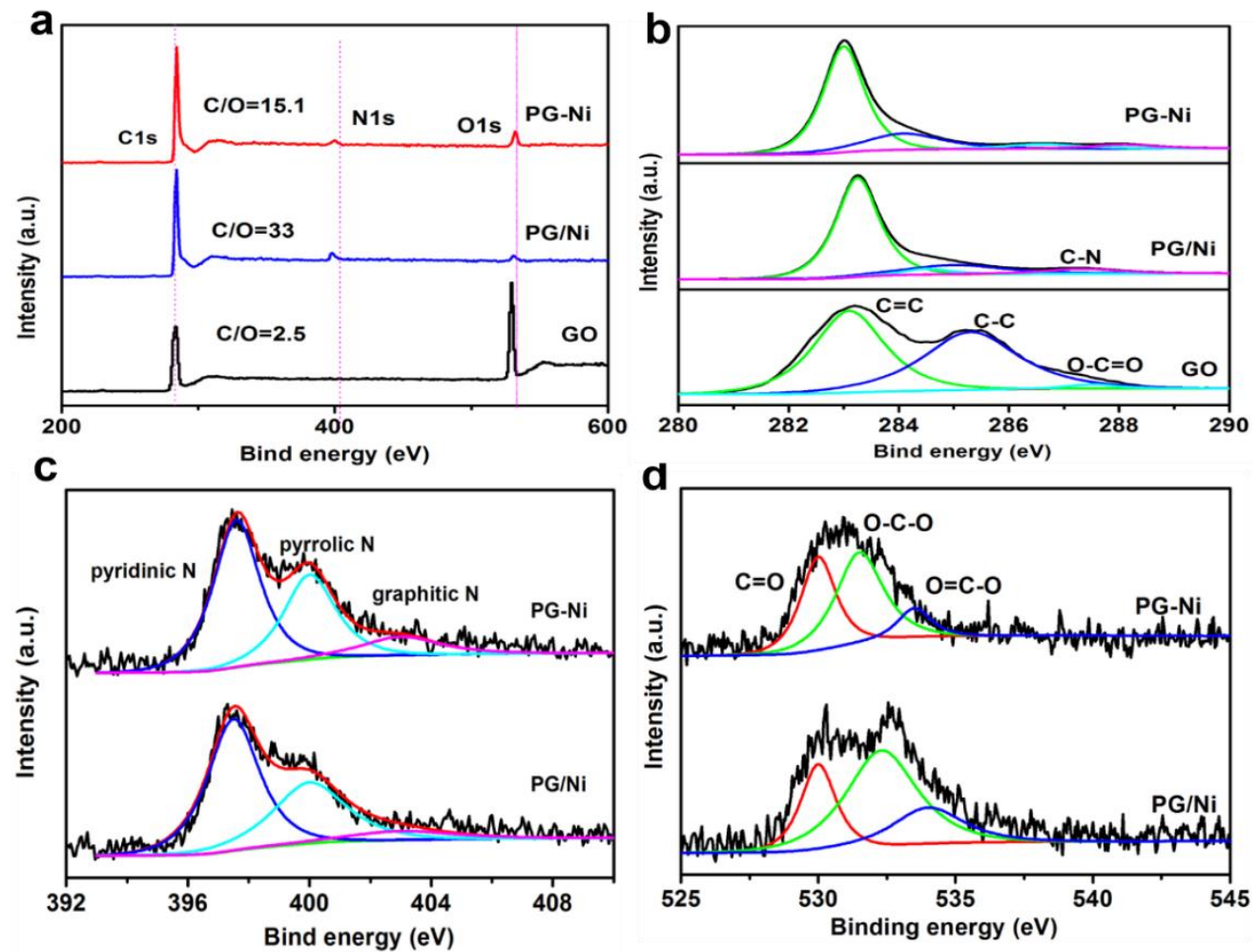

Fig. 2. XPS results of GO, PG/Ni and PG-Ni. a. survey scan; b. C1s spectra; c. N 1s XPS spectra of PG/Ni and PG-Ni; d. O 1s XPS spectra of PG/Ni and PG-Ni.

One of the important features of this method is the $\mathrm{N}$-doping of graphene in $\mathrm{N}_{2}$ gas during the pore generation process. In order to prove the N-doping of $\mathrm{PG}$, we have performed XPS analysis and TEM element mapping of PG-Ni and PG-Co. The both XPS and TEM mapping suggest the presence of nitrogen element (Fig.2 and S2), suggesting the successful nitrogen doping into PG. The XPS and the element mapping shows that for PG-Ni has a $\mathrm{N}$ doping atomic ratio of $4.44 \%$, while that of PG-Co is only $1.32 \%$, suggesting the $\mathrm{N}$ doping content would be controlled by the different metal salts. (Fig. 2 and Fig. S3). The N1s spectra of both PG-Ni and PG-Co shows the pyridinic, pyrrolic and graphitic nitrogen,. Compare the N1s spectra of PG-Ni and PG-Co, it is found that for PG-Ni, there are $64 \%$ of pyridinic nitrogen, $28 \%$ pyrrolic nitrogen, and 
$8 \%$ of graphitic nitrogen, however, for PG-CO, the pyridinic nitrogen only takes $49.9 \%$, with $28.7 \%$ pyrrolic nitrogen and an increased graphitic nitrogen ratio of $21.4 \%$, indicating the choice of different etchants can affect the nitrogen doping content and nitrogen doping morphology. It is worth noting that pyridinic configuration is favored for supercapacitor[35-38], this further encourage us to explore the electrochemical performance of the PG-Ni and PG-Co in the supercapacitor. Dut to the chemical interness, the $\mathrm{N}_{2}$ gas is not frequently used for $\mathrm{N}$-doping, but here we observed $\mathrm{N}$ doping in $\mathrm{PG}$ nanosheets by $\mathrm{N}_{2}$. This is quite surprising, we speculate the reason is, the $\mathrm{Ni}$ and Co particle formed in the heating process can used as the catalysts, when the sample was heated to $800{ }^{\circ} \mathrm{C}$, the $\mathrm{N}_{2}$ are decomposed, and during the pore generation process, the freshly formed edges and defect sites further decrease the energy barrier for $\mathrm{N}$-doping $[39,40]$. A higher $\mathrm{N}$ content in PG-Ni could be explained by that, due to the fine particle size, a higher effective surface area and therefore more active sites during the nitrogen doping process are available to enable a high $\mathrm{N}$-doping content in PG-Ni.

To exclude the N-doping is related to impurities, we conducted the thermal treatment of GO nanosheets with metal salt in Ar atomosphere, XPS analysis shows no doping effects in the resulting graphene nanosheets (Fig.S4), therefore, further confirming the $\mathrm{N}$-doping is originated from $\mathrm{N}_{2}$ gas. Furthermore, we changed the $\mathrm{N}_{2}$ to urea as nitrogen doping agent, the resulting graphene nanosheets shows 6.12 at.\% N-doping (Fig.S5 and Tab S1), which is higher than PG-Ni, however, the nitrogen configuration is mainly pyrrolic nitrogen which is not favored for the high electrochemical capacitance(是不是 
要引用个 SI 图, reference 啥的).
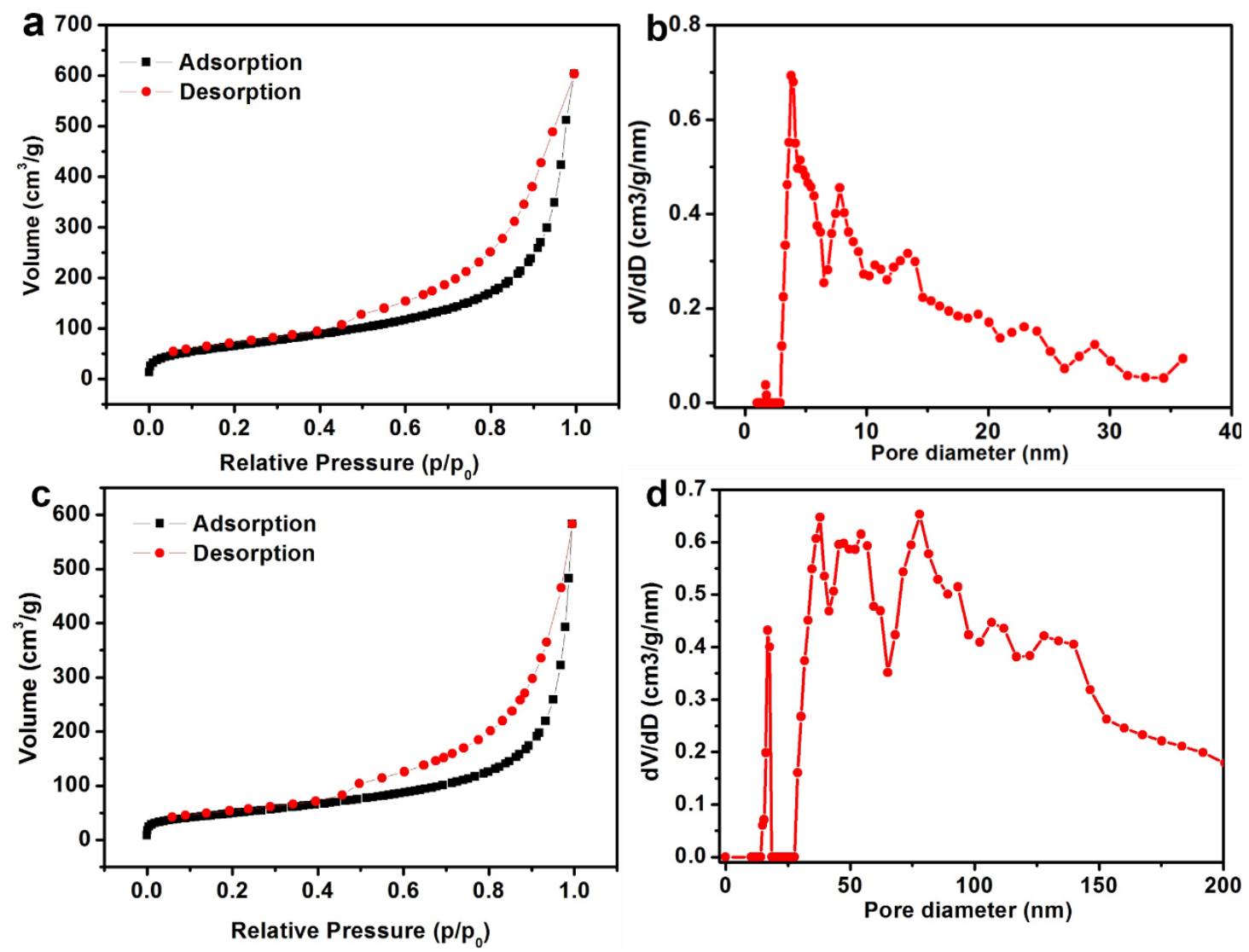

Fig. 3. a. Nitrogen adsorption-desorption isotherms and b. pore size distributions of PG-Ni; c.

Nitrogen adsorption-desorption isotherms and d. pore size distributions of PG-Co.

The pore generation and N-doping will introduce abundant pores and active sites for energy storage applications, we therefore conducted the BET measurement to study the surface area and pore structure of PG materials. As shown in Fig. 3, the specific surface area of Ni-PG sample is about $388 \mathrm{~m}^{2} / \mathrm{g}$, which is higher than Co-PG sample $\left(244 \mathrm{~m}^{2} / \mathrm{g}\right)$ andRGO $\left(115 \mathrm{~m}^{2} / \mathrm{g}\right)$ (Fig.S6). Obviously, this shows that the porous structure could effectively increase the surface area of the graphene nanosheets. Further analysis enables us to derive the information of pore structures of Ni-PG and Co-PG. The pore volume of PG-Ni is $0.671 \mathrm{~m}^{3} / \mathrm{g}$ and average pore width is about $20 \mathrm{~nm}$ while the total 
pore volume of PG-Co is $0.723 \mathrm{~m}^{3} / \mathrm{g}$ and average pore width is about $90 \mathrm{~nm}$, which is consistent with the SEM observation[41].

\subsection{Electrochemical properties of the supercapacitor}
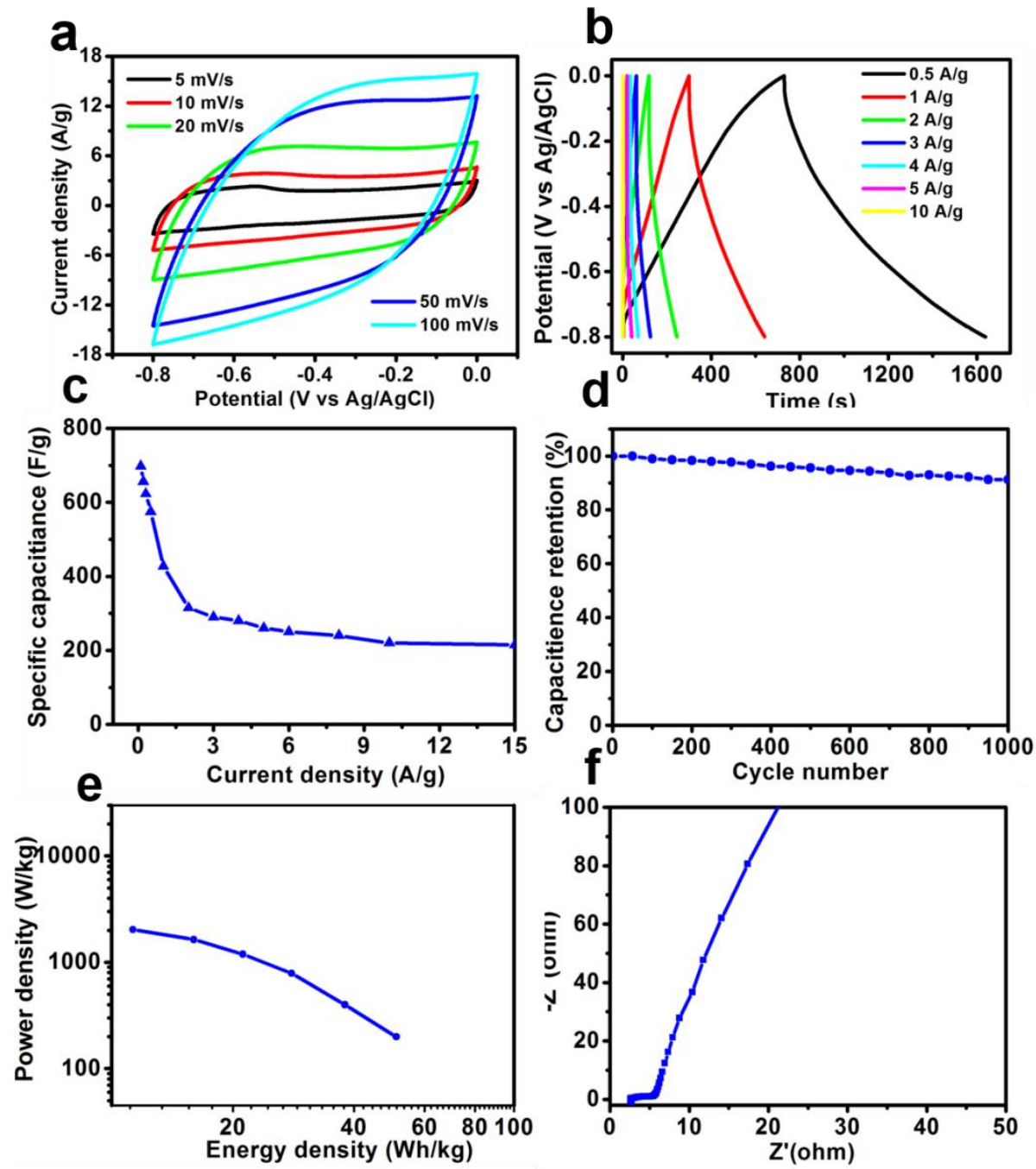

Fig. 4. Capacitive performance of PG-Ni in KOH electrolyte. a. CV curves at 5, 10, 20, 50 and 100 $\mathrm{mV} / \mathrm{s}$ in three-electrode system; b. GCD curves; c. specific capacitance at different current densities; d. cycle stability test; e. Bode plot; f. Ragone plots. 


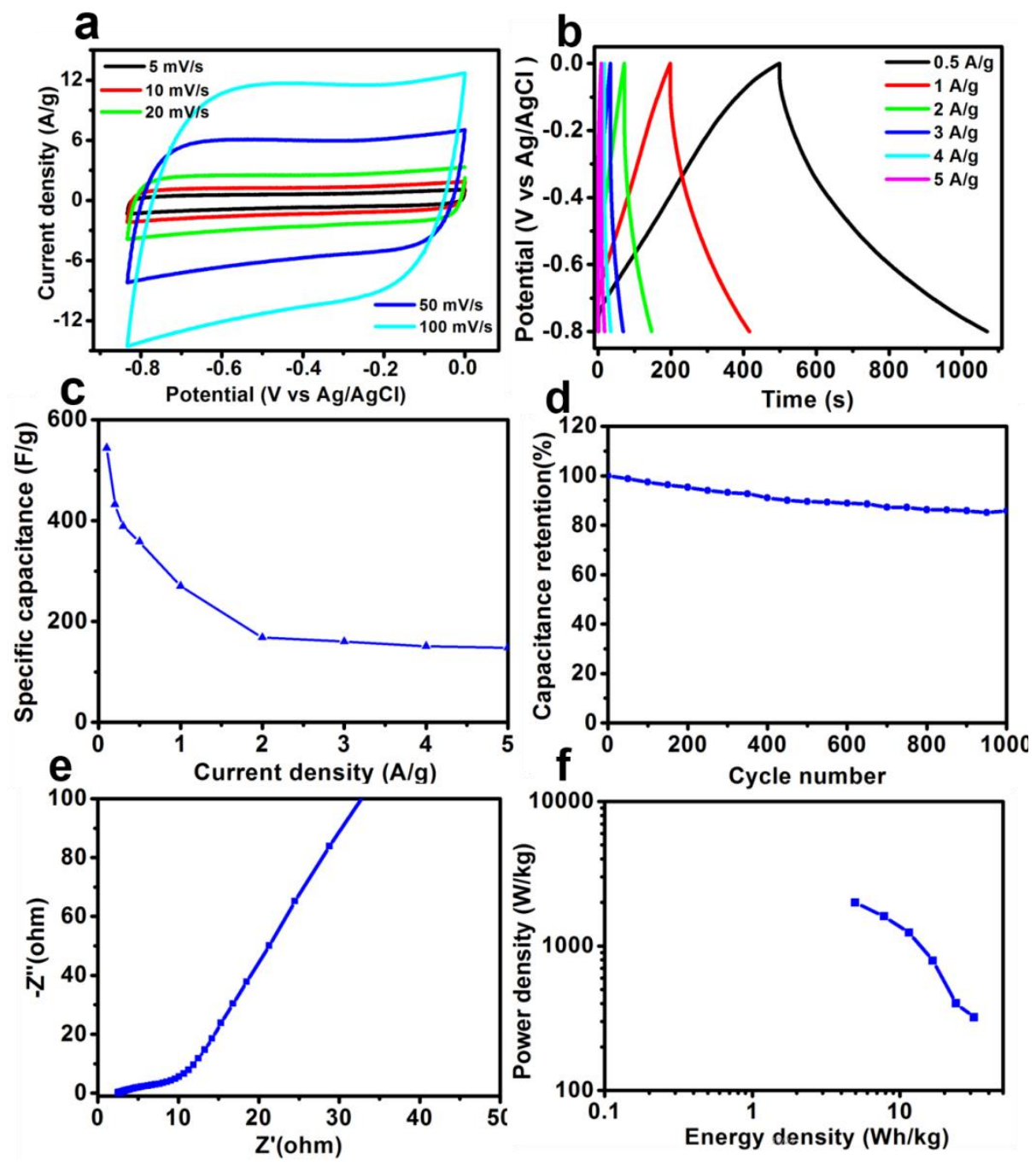

Fig. 5. Capacitive performance of PG-Co in $\mathrm{KOH}$ electrolyte. a. CV curves at 5, 10, 20, 50 and 100 $\mathrm{mV} / \mathrm{s}$ in three-electrode system; b. GCD curves; c. specific capacitance at different current densities; d. cycle stability test; e. Bode plot; f. Ragone plots.

We then assembled the PG based supercapacitor and conducted a serious of electrochemical measurements. We found that, the rGO nanosheets only show a specific capacitance of $110 \mathrm{~F} / \mathrm{g}$, and the PG nanosheets without N-doping show a capacitance of $230 \mathrm{~F} / \mathrm{g}$ (Fig.S7), This indicates that the porous structure can improve the performance for supercapacitor. We then further examined the two N-doping PG (PG$\mathrm{Ni}$ and PG-Co) for supercapacitor electrodes, and we found thatthe specific capacities 
for PG-Ni and PG-Co are 575 and $350 \mathrm{~F} / \mathrm{g}$ at $0.5 \mathrm{~A} / \mathrm{g}$, respectively, which is much higher than that of the PG without N-doping obtained (Fig. 4 and 5), suggesting the N-doping are effective for the high electrochemical capacitance. Comparing with pure PG without $\mathrm{N}$-doping, there are two peaks at $-0.53 \mathrm{~V}$ and $-0.67 \mathrm{~V}$ for PG-Ni sample attributed to the redox reactions of $\mathrm{N}$-doping on the surface suggesting capacitive improvement is attributed to the combination of the electrical double-layer capacitance and the Faradaic capacitance. To further understand the N-doping on the capacitance of supercapacitor, we studied the PG nanosheets doped with urea for their supercapacitor application. We found the urea doped PG, though shows a higher N-doping content ( 6.12 at\%), only has a capacitance of $180 \mathrm{~F} / \mathrm{g}$ at $0.5 \mathrm{~A} / \mathrm{g}$, which is much smaller than that of PG-Ni (Fig.S9). Considering that the major $\mathrm{N}$ doping configurations in urea treated sample is pyrrolic nitrogen, while, for PG-Ni, that is pyridine nitrogen, this suggests, the doped nitrogen configuration might play an important role in electrochemical capacitance. Since the pyridine nitrogen atom on the substrate has strong bonding with the cations in the solution, the cation density on the surface of the electrode is greatly improved, thereby improving the effective specific surface area of PG materials, which in turn increases the specific capacitance of PG. These can indicate that the proper N-doping structure is very important for performance of supercapacitor. Fig. $4 \mathrm{~d}$ and $5 \mathrm{~d}$ showed the long-term cyclic stability of the PG-Ni and PG-Co based supercapacitor by calculating the capacitance value from the CVs over 1000 at $2 \mathrm{~A} / \mathrm{g}$. As shown in Fig. 4d, the PG-Ni remained $93.6 \%$ of its initial capacitance after 1000 cycles, showing good cycling stability. Fig. $4 \mathrm{f}$ and $5 \mathrm{f}$ are the Ragone plots, demonstrating the connection 
between the energy density (E) and power density (P),the PG-Ni exhibited an energy density of about $11 \mathrm{Wh} / \mathrm{kg}$ at a power density of $2000 \mathrm{~W} / \mathrm{kg}$, and retains a remarkable energy density of $50 \mathrm{Wh} / \mathrm{kg}$ at a high power density of $200 \mathrm{~W} \mathrm{~kg}^{-1}$. This is higher than most of previously reported work (Tab S3).Furthermore, the energy density of PG-Ni held over greater than $20 \%$ of its initial value when the consistent current density expanded from 0.5 to $5 \mathrm{~A} / \mathrm{g}$. These experimental confirmed that the high performance to enhanced ion diffusion channels due to the pores on the graphene surface. And on the other hand is the introduction of nitrogen atoms. The specific capacitance of $\mathrm{N}$ doping graphene and the structure distribution of nitrogen in graphene are related[48]. We have further studied the kinetics of ion transport using EIS with the frequency ranging from $100 \mathrm{kHz}$ to $10 \mathrm{mHz}$. Fig. 4e and 5e showed the Nyquist plots of PG series sample. At the low frequency region the PG samples electrode exhibited nearly vertical line indicating high specific capacitance and fast charge transfer. The Nyquist plots are simulated by ZView software, that the Rct of the PG-Ni supercapacitor was estimated to be $0.25 \Omega$, which was much smaller than $0.98 \Omega$ (Tab. S2) for the rGO and $0.4 \Omega$ for the PG-Co supercapacitor, the simulated values of Rst were $0.95 \Omega, 1.98 \Omega$ for PG-Co, and $5.98 \Omega$ for rGO. The smaller Rct demonstrated more proficient charge transfer and ion penetration that prompt a great rate performance.

\section{Conclusion}

In summary, we have fabricated the N-doping PG by the one-step synthesis using environment friendly $\mathrm{N}_{2}$ gas as doping agent by a thermal treatment of metal salt 
infiltrated graphene nanosheetsin $\mathrm{N}_{2}$ atmosphere. We found that the pore structure could be tuned by different metal ions, and the N-doping content and structure could be controlled by the etching and doping agent. Furthermore, the N-doped PG nanosheets were examined for their applications in supercapacitors, it is found that, comparing to the pure rGO, and other N-doped PG nanosheets, the N-doped PG nanosheets obtained by Ni nanoparticle etching shows the highest supercapacitance of $575 \mathrm{~F} / \mathrm{g}$, which could be explained by the fine pore size, high pore density and pyridine nitrogen dominated nitrogen doping configuration. Our study shows that the pore size and N-doping have synergetic effects on improving the electrochemical capacitance of the graphene materials, bring together with the good processability, controllability of the proposed

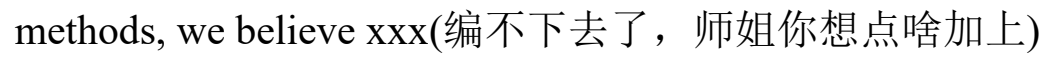

\section{Acknowledgements}

This work was supported by project ZR2019MB034. Project ZR2019MB034 was supported by Shandong Provincial Natural Science Foundation. This work was supported by National Natural Science Foundation of China (No. 21576159, 51907110).

[1] H. Huang, C. Lei, G. Luo, Z. Cheng, G. Li, S. Tang, Y. Du, Facile synthesis of nitrogeN-doping graphene on Ni foam for high-performance supercapacitors, Journal of Materials Science 51(13) (2016) 63486356.

[2] P. lamprasertkun, A. Krittayavathananon, M. Sawangphruk, N-doped reduced graphene oxide aerogel coated on carboxyl-modified carbon fiber paper for high-performance ionic-liquid supercapacitors, Carbon 102 (2016) 455-461.

[3] Université Paul Sabatier, Materials for electrochemical capacitors, Nanoscience and Technology, (2009) 320-329.

[4] Yanwu Zhu, 1 Shanthi Murali, 1 Meryl D. Stoller, 1 K. J. Ganesh, 1 Weiwei Cai, 1 Paulo J. Ferreira, Carbon-Based Supercapacitors Produced by Activation of Graphene, Science (2011) 1537-1541.

[5] L.L. Zhang, R. Zhou, X.S. Zhao, Graphene-based materials as supercapacitor electrodes, Journal of Materials Chemistry 20(29) (2010).

[6] A.K. Geim, K.S. Novoselov, The rise of graphene, Nat. Mater. 6 (2007) 183-191. 
[7] J. Xia, F. Chen, J. Li, N. Tao, Measurement of the quantum capacitance of graphene, Nat Nanotechnol 4(8) (2009) 505-9.

[8] Q. Ke, J. Wang, Graphene-based materials for supercapacitor electrodes - A review, Journal of Materiomics 2(1) (2016) 37-54.

[9] A. Bakandritsos, P. Jakubec, M. Pykal, M. Otyepka, Covalently functionalized graphene as a supercapacitor electrode material, FlatChem 13 (2019) 25-33.

[10] Z. Chu, R. He, X. Zhang, Z. Jiang, H. Cheng, Y. Wang, X. Li, Fabrication of porous SiCy (core)/C (shell) fibres using a hybrid precursor of polycarbosilane and pitch, Carbon 48(7) (2010) 2115-2118.

[11] S. Stankovich, D.A. Dikin, G.H. Dommett, K.M. Kohlhaas, E.J. Zimney, E.A. Stach, R.D. Piner, S.T. Nguyen, R.S. Ruoff, Graphene-based composite materials, Nature 442(7100) (2006) 282-6.

[12] C. Ma, Y. Zhao, Y. Li, A facile solution-free etching preparation of porous graphene nanosheets with high performances for lithium storage, Chemical Engineering Journal 320 (2017) 283-289.

[13] H. Duan, T. Yan, G. Chen, J. Zhang, L. Shi, D. Zhang, A facile strategy for the fast construction of porous graphene frameworks and their enhanced electrosorption performance, Chem Commun (Camb) 53(54) (2017) 7465-7468.

[14] A. Amiri, M. Shanbedi, G. Ahmadi, H. Eshghi, S.N. Kazi, B.T. Chew, M. Savari, M.N.M. Zubir, Mass production of highly-porous graphene for high-performance supercapacitors, Scientific Reports 6(1) (2016).

[15] X. Zhang, H. Zhang, C. Li, K. Wang, X. Sun, Y. Ma, Recent advances in porous graphene materials for supercapacitor applications, RSC Adv. 4(86) (2014) 45862-45884.

[16] J.H. Jeong, G.-W. Lee, Y.H. Kim, Y.J. Choi, K.C. Roh, K.-B. Kim, A holey graphene-based hybrid supercapacitor, Chemical Engineering Journal 378 (2019).

[17] Y. Xu, Z. Lin, X. Zhong, X. Huang, N.O. Weiss, Y. Huang, X. Duan, Holey graphene frameworks for highly efficient capacitive energy storage, Nat Commun 5 (2014) 4554.

[18] S. Dou, L. Tao, J. Huo, S. Wang, L. Dai, Etched and doped Co9S8/graphene hybrid for oxygen electrocatalysis, Energy \& Environmental Science 9(4) (2016) 1320-1326.

[19] J. Lee, K. Kim, W.I. Park, B.H. Kim, J.H. Park, T.H. Kim, S. Bong, C.H. Kim, G. Chae, M. Jun, Y. Hwang, Y.S. Jung, S. Jeon, Uniform graphene quantum dots patterned from self-assembled silica nanodots, Nano Lett 12(12) (2012) 6078-83.

[20] D.-P. Yang, X. Wang, X. Guo, X. Zhi, K. Wang, C. Li, G. Huang, G. Shen, Y. Mei, D. Cui, UV/O3 Generated Graphene Nanomesh: Formation Mechanism, Properties, and FET Studies, The Journal of Physical Chemistry C 118(1) (2013) 725-731.

[21] T.H. Han, Y.K. Huang, A.T. Tan, V.P. Dravid, J. Huang, Steam etched porous graphene oxide network for chemical sensing, J Am Chem Soc 133(39) (2011) 15264-7.

[22] J. Bai, X. Zhong, S. Jiang, Y. Huang, X. Duan, Graphene nanomesh, Nat Nanotechnol 5(3) (2010) 190-4.

[23] M. Zhou, T. Tian, X. Li, X. Sun, J. Zhang, Y. Chen, P. Cui, J. Tang, L.-C. Qin, Supercapacitance of chemically converted graphene with composite pores, Chemical Physics Letters 581 (2013) 64-69. [24] Y. Zhu, S. Murali, M.D. Stoller, K.J. Ganesh, W. Cai, P.J. Ferreira, A. Pirkle, R.M. Wallace, K.A. Cychosz, M. Thommes, D. Su, E.A. Stach, R.S. Ruoff, Carbon-based supercapacitors produced by activation of graphene, Science 332(6037) (2011) 1537-41.

[25] M. Yang, Z. Zhou, Recent Breakthroughs in Supercapacitors Boosted by Nitrogen-Rich Porous Carbon Materials, Adv Sci (Weinh) 4(8) (2017) 1600408.

[26] D. Hulicova-Jurcakova, M. Seredych, G.Q. Lu, T.J. Bandosz, Combined Effect of Nitrogen- and 
Oxygen-Containing Functional Groups of Microporous Activated Carbon on its Electrochemical Performance in Supercapacitors, Advanced Functional Materials 19(3) (2009) 438-447.

[27] P. Bharathidasan, M.B. Idris, D.-W. Kim, S.R. Sivakkumar, S. Devaraj, Enhanced capacitance properties of nitrogen doped reduced graphene oxide obtained by simultaneous reduction and nitrogen doping, FlatChem 11 (2018) 24-31.

[28] H. Wang, T. Maiyalagan, X. Wang, Review on Recent Progress in Nitrogen-Doped Graphene: Synthesis, Characterization, and Its Potential Applications, ACS Catalysis 2(5) (2012) 781-794.

[29] Lei Wang, Chungui Tian, Huan Wang, Yuguang Ma, Baoli Wang, and Honggang Fu, Mass Production of Graphene via an in Situ Self-Generating Template Route and Its Promoted Activity as Electrocatalytic Support for Methanol Electroxidization, J. Phys. Chem. C ( 2010), 114, 8727-8733. [30] Leonardo C. Campos, Vitor R. Manfrinato, Javier D. Sanchez-Yamagishi, Jing Kong, Pablo JarilloHerrero, Anisotropic Etching and Nanoribbon Formation in Single-Layer Graphene, Nano Lett.(2009) 9, 7, 2600-2604.

[31] L. Ci, Z. Xu, L. Wang, W. Gao, F. Ding, K.F. Kelly, B.I. Yakobson, P.M. Ajayan, Controlled nanocutting of graphene, Nano Research 1(2) (2008) 116-122.

[32] Quentin M. Ramasse, Recep Zan, Ursel Bangert, Danil W. Boukhvalov, Young-Woo Son, Konstantin S. Novoselov, Direct Experimental Evidence of Metal-Mediated Etching of Suspended Graphene, ACS Nano(2012) 6, 5, 4063-4071.

[33] H. Cao, X. Zhou, C. Zheng, Z. Liu, Metal etching method for preparing porous graphene as high performance anode material for lithium-ion batteries, Carbon 89 (2015) 41-46.

[34] Jinping Zhao, Songfeng Pei, Wencai Ren, Libo Gao, and Hui-Ming Cheng, Efficient Preparation of Large-Area Graphene Oxide Sheets for Transparent Conductive Films, ACS Nano(2010) 4, 9, 5245-5252

[35] P. Xu, Q. Gao, L. Ma, Z. Li, H. Zhang, H. Xiao, X. Liang, T. Zhang, X. Tian, C. Liu, A high surface area $\mathrm{N}$-doped holey graphene aerogel with low charge transfer resistance as high performance electrode of non-flammable thermostable supercapacitors, Carbon 149 (2019) 452-461.

[36] W. Zhang, C. Xu, C. Ma, G. Li, Y. Wang, K. Zhang, F. Li, C. Liu, H.M. Cheng, Y. Du, N. Tang, W. Ren, Nitrogen-Superdoped 3D Graphene Networks for High-Performance Supercapacitors, Adv Mater 29(36) (2017).

[37] J.H. Lee, S.H. Kwon, S. Kwon, M. Cho, K.H. Kim, T.H. Han, S.G. Lee, Tunable Electronic Properties of Nitrogen and Sulfur Doped Graphene: Density Functional Theory Approach, Nanomaterials (Basel)

9(2) (2019).

[38] K. Akada, T.-o. Terasawa, G. Imamura, S. Obata, K. Saiki, Control of work function of graphene by plasma assisted nitrogen doping, Applied Physics Letters 104(13) (2014).

[39] F. Bourquard, Y. Bleu, A.S. Loir, B. Caja-Munoz, J. Avila, M.C. Asensio, G. Raimondi, M. Shokouhi, I. Rassas, C. Farre, C. Chaix, V. Barnier, N. Jaffrezic-Renault, F. Garrelie, C. Donnet, Electroanalytical Performance of Nitrogen-Doped Graphene Films Processed in One Step by Pulsed Laser Deposition Directly Coupled with Thermal Annealing, Materials (Basel) 12(4) (2019).

[40] X. Deng, B. Zhao, L. Zhu, Z. Shao, Molten salt synthesis of nitrogen-doped carbon with hierarchical pore structures for use as high-performance electrodes in supercapacitors, Carbon 93 (2015) 48-58.

[41] Y. Lin, K.A. Watson, J.W. Kim, D.W. Baggett, D.C. Working, J.W. Connell, Bulk preparation of holey graphene via controlled catalytic oxidation, Nanoscale 5(17) (2013) 7814-24.

[42] W. Chen, Z. Hu, Y. Yang, X. Wang, Y. He, Y. Xie, C. Zhu, Y. Zhang, L. Lv, Controlling synthesis of nitrogen-doped hierarchical porous graphene-like carbon with coral flower structure for highperformance supercapacitors, Ionics 25(11) (2019) 5429-5443. 
[43] Elzbieta Frackowiak, Francois Beguin, Carbon materials for the electrochemical storage of energy in capacitors, Carbon 39 (2001) 937 - 950.

[44] P.-Z. Cheng, H. Teng, Electrochemical responses from surface oxides present on HNO3-treated carbons, Carbon 41(11) (2003) 2057-2063.

[45] P. Sazama, J. Pastvova, C. Rizescu, A. Tirsoaga, V.I. Parvulescu, H. Garcia, L. Kobera, J. Seidel, J. Rathousky, P. Klein, I. Jirka, J. Moravkova, V. Blechta, Catalytic Properties of 3D Graphene-Like Microporous Carbons Synthesized in a Zeolite Template, ACS Catalysis 8(3) (2018) 1779-1789.

[46] H. Jiang, P.S. Lee, C. Li, 3D carbon based nanostructures for advanced supercapacitors, Energy Environ. Sci. 6(1) (2013) 41-53.

[47] L. Sun, L. Wang, C. Tian, T. Tan, Y. Xie, K. Shi, M. Li, H. Fu, Nitrogen-doped graphene with high nitrogen level via a one-step hydrothermal reaction of graphene oxide with urea for superior capacitive energy storage, RSC Advances 2(10) (2012).

[48] Y. Deng, Y. Xie, K. Zou, X. Ji, Review on recent advances in nitrogen-doped carbons: preparations and applications in supercapacitors, Journal of Materials Chemistry A 4(4) (2016) 1144-1173. 